\title{
Uncertainty associated with form assessment in coordinate metrology
}

\author{
A.B. Forbes* \\ National Physical Laboratory, Teddington, Middlesex, UK
}

Received: 12 November 2012 / Accepted: 22 November 2012

\begin{abstract}
In this paper, we describe techniques for evaluating the uncertainties associated with the assessment of form error, i.e., the departure from ideal geometry of a manufactured part, in coordinate metrology. The techniques take into account measurement uncertainty, sampling effects due to the fact that the form error is determined from a finite set of coordinate data points, and the spatial correlation of the form errors. The techniques are designed to be practical, without the need for complex computation.
\end{abstract}

Keywords: Form error; Gaussian processes; uncertainty

\section{Introduction}

Form error refers to the departure from ideal geometry for a manufactured part. In coordinate metrology, form error is inferred from the analysis of coordinate data representing points $X=\left\{\boldsymbol{x}_{i}, i=1, \ldots, m\right\}$ on the surface of the manufactured part. We assume the ideal geometry can be represented by a parametric surface $\boldsymbol{u} \mapsto \mathcal{S}(\boldsymbol{u}, \boldsymbol{b})$ where $\boldsymbol{u}$ are the patch parameters determining the location of a point on the surface and $\boldsymbol{b}$ are parameters determining the position, size and shape of the surface. For example, a circle with centre coordinates $\left(x_{0}, y_{0}\right)^{\mathrm{T}}$ and radius $r_{0}$ is specified by

$$
u \mapsto \mathcal{C}(u, \boldsymbol{b})=\left(x_{0}+r_{0} \cos u, y_{0}+r_{0} \sin u\right)^{\mathrm{T}},
$$

where $\boldsymbol{b}=\left(x_{0}, y_{0}, r_{0}\right)^{\mathrm{T}}$. Given data points $X$, the best fit parameters of the surface is found by minimising some aggregate measure involving the distances $d\left(\boldsymbol{x}_{i}, \boldsymbol{b}\right)$ where $d(\boldsymbol{x}, \boldsymbol{b})$ is the (signed) orthogonal distance from the point $\boldsymbol{x}$ to the surface $\mathcal{S}(\boldsymbol{u}, \boldsymbol{b})$ [1-13]. For example, Gaussian or Chebyshev criteria are often used:

$$
\min _{\boldsymbol{b}} \sum_{i} d^{2}\left(\boldsymbol{x}_{i}, \boldsymbol{b}\right), \quad \min _{\boldsymbol{b}} \max _{i}\left|d\left(\boldsymbol{x}_{i}, \boldsymbol{b}\right)\right| .
$$

For the case of a circle,

$$
d(\boldsymbol{x}, \boldsymbol{b})=\left(\left(x-x_{0}\right)^{2}+\left(y-y_{0}\right)^{2}\right)^{1 / 2}-r_{0} .
$$

For other standard geometric shapes such as a plane, sphere, cylinder and cone, the distance function can be determined analytically. For free form surfaces numerical methods are usually required to evaluate the distance function.

^ Correspondence: Alistair.Forbes@npl.co.uk
Once the fitted surface has been determined, the residual distances $d_{i}=d\left(\boldsymbol{x}_{i}, \boldsymbol{b}\right)$ at the solution estimate the departure $f_{i}$ from ideal geometry of the surface near $\boldsymbol{x}_{i}$ and the form error $F_{E}$ for the complete surface is estimated by $F_{E}=\max _{i} d_{i}-\min _{i} d_{i}$. We wish to evaluate the uncertainty associated with this estimate taking into account two influence factors. The first is the fact that the measured values will be subject to random and possibly systematic effects that will tend to lead to an overestimate of the form error. For example, measuring a perfect geometry with a noisy instrument will always lead to a non-zero estimate of the form error. The second influence factor relates to the sampling effect, reflecting the fact that the form error is determined from a finite, discrete sample of the surface and is therefore likely to underestimate the form error of the complete surface.

The rest of this paper is organised as follows. In Section 2, we describe analysis methods based on a simple model of form error using rectangular distributions while in Section 3 we consider models that attempt to take into account the spatial correlation of form error. Section 4 discusses the approaches in Sections 2 and 3 and how they can be extended. Our concluding remarks are given in Section 5 .

\section{Models of form error based on rectangular distributions}

In this section, we assume that the form error $f_{i}$ associated with a point $\boldsymbol{s}_{i}=\mathcal{S}\left(\boldsymbol{u}_{i}, \boldsymbol{b}\right)$ on the ideal surface is modelled as belonging to a rectangular distribution whose parameters are related to the form error $F_{E}$ for the complete surface. The goal is to make inferences about the form error on the basis of the observed estimates of the form error. 


\subsection{Symmetric rectangular distribution}

Suppose that $\boldsymbol{f}=\left(f_{1}, \ldots, f_{m}\right)^{\mathrm{T}}$ are random, independent draws from a rectangular distribution $\mathrm{R}(-F, F)$,

$$
f_{i} \in \mathrm{R}(-F, F) .
$$

Having observed, $f_{i}, i=1, \ldots, m$, what can be said about $F$ ? Bayesian inference [14-17] can be used to provide an answer to this question. The density associated with the probability of observing $\boldsymbol{f}$, given $F$, is given by

$$
p(\boldsymbol{f} \mid F)=\frac{1}{2^{m}} \frac{1}{F^{m}},
$$

if $F \geq F_{0}=\max _{i}\left|f_{i}\right|$, and zero otherwise. Given a prior distribution $p(F)$ for $F$ and applying Bayes' theorem the posterior distribution for $F$, given $\boldsymbol{f}$, is such that

$$
p(F \mid \boldsymbol{f}) \propto p(\boldsymbol{f} \mid F) p(F) .
$$

Treating $F$ as a scale parameter, a suitable noninformative prior for $F$ is $p(F) \propto 1 / F$, and with this choice

$$
p(F \mid \boldsymbol{f}) \propto \frac{1}{F^{m+1}}, \quad F \geq F_{0}
$$

and is zero elsewhere. In fact $p(F \mid \boldsymbol{f})$ has a Pareto distribution [18]

$$
p\left(F \mid F_{0}, m\right)=\frac{m F_{0}^{m}}{F^{m+1}},
$$

with mean

$$
\mathrm{E}\left(F \mid F_{0}, m\right)=\frac{m F_{0}}{m-1}, \quad m>1,
$$

and variance

$$
\operatorname{var}\left(F \mid F_{0}, m\right)=\left(\frac{F_{0}}{m-1}\right)^{2} \frac{m}{m-2}, \quad m>2 .
$$

The quartiles of this distribution are such that if $f=$ $F_{0} / \alpha^{1 / m}$, then

$$
\operatorname{Pr}\left(F \geq f \mid F_{0}, m\right)=\alpha .
$$

Example calculations are given in Table 1. For example, if $m=8$ and a value for $F_{0}=0.010 \mathrm{~mm}$ is observed, then there is a $10 \%$ probability that $F$ is greater than $0.01 \times 1.33=0.013 \mathrm{~mm}$, according to this model.

\subsubsection{Incorporating measurement uncertainty}

The above calculations assume that the observed residual distances $d_{i}$ are accurate estimates of the form errors $f_{i}$ associated with the surface. Suppose now that

$$
d_{i}=f_{i}+\epsilon_{i},
$$

with

$$
f_{i} \in \mathrm{R}(-F, F), \quad \epsilon_{i} \in \mathrm{N}\left(0, \sigma_{M}^{2}\right),
$$

\begin{tabular}{ccccc}
\hline$m$ & $\mu$ & $Q_{50}$ & $Q_{90}$ & $Q_{95}$ \\
\hline 2 & 2.00 & 1.41 & 3.16 & 4.47 \\
3 & 1.50 & 1.26 & 2.15 & 2.71 \\
4 & 1.33 & 1.19 & 1.78 & 2.11 \\
6 & 1.20 & 1.12 & 1.47 & 1.65 \\
8 & 1.14 & 1.09 & 1.33 & 1.45 \\
\hline 10 & 1.11 & 1.07 & 1.26 & 1.35 \\
15 & 1.07 & 1.05 & 1.17 & 1.22 \\
20 & 1.05 & 1.04 & 1.12 & 1.16 \\
30 & 1.03 & 1.02 & 1.08 & 1.11 \\
50 & 1.02 & 1.01 & 1.05 & 1.06 \\
\hline
\end{tabular}

Table 1. Mean and specified quartiles associated with the Pareto distribution $p\left(F \mid F_{0}\right)$ in (2) for $F_{0}=1$ and different values of $m$.

reflecting random effects associated with the measured values drawn from a Gaussian distribution with standard deviation $\sigma_{M}$. What now can be said about $F$, given that $\boldsymbol{d}=\left(d_{1}, \ldots, d_{m}\right)^{\mathrm{T}}$ has been observed? A quick estimate of $F$ can be derived as follows. Since the variance of the rectangular distribution $\mathrm{R}(-F, F)$ is $F^{2} / 3$, then according to the model (and again assuming independence) the $d_{i}$ are draws from a distribution with variance

$$
\frac{F^{2}}{3}+\sigma_{M}^{2}
$$

If $s_{D}$ is the standard deviation of the residual distances,

$$
s_{D}^{2}=\frac{1}{m-1} \sum_{i}\left(d_{i}-\bar{d}\right)^{2}, \quad \bar{d}=\frac{1}{m} \sum_{i} d_{i},
$$

then $F$ is estimated by $\hat{F}$ where

$$
\hat{F}^{2}=3\left(s_{D}^{2}-\sigma_{M}^{2}\right)
$$

if $s_{D}>\sigma_{M}$ and is zero otherwise.

An analysis based on Bayesian inference is also possible. We have

$$
p\left(d_{i} \mid F\right)=\int p\left(d_{i}, f_{i} \mid F\right) d f_{i},
$$

and

$$
p\left(d_{i}, f_{i} \mid F\right)=p\left(d_{i} \mid f_{i}, F\right) p\left(f_{i} \mid F\right) .
$$

The first density on the right is that associated with the Gaussian distribution $\mathrm{N}\left(f_{i}, \sigma_{M}^{2}\right)$ and the second, that associated with $\mathrm{R}(-F, F)$. As a result,

$$
p\left(d_{i} \mid F\right)=\frac{1}{2 F} \int_{-F}^{F} p\left(d_{i} \mid f, \sigma_{M}\right) d f .
$$

This integral can be evaluated using the error function erf: if $p(x \mid \mu, \sigma)$ is the density associated with the Gaussian distribution $\mathrm{N}\left(\mu, \sigma^{2}\right)$ then

$$
\begin{aligned}
I(\mu, \sigma, A, B) & =\int_{A}^{B} p(x \mid \mu, \sigma) d x, \\
& =\frac{1}{2} \operatorname{erf}\left(\frac{B-\mu}{\sqrt{2} \sigma}\right)-\frac{1}{2} \operatorname{erf}\left(\frac{A-\mu}{\sqrt{2} \sigma}\right) .
\end{aligned}
$$




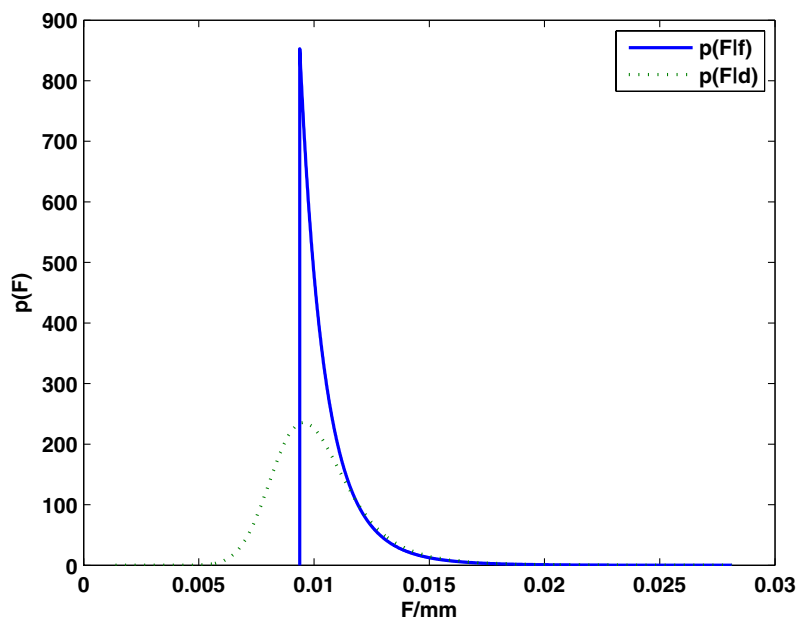

Fig. 1. Posterior densities $p(F \mid \boldsymbol{f})$ and $p(F \mid \boldsymbol{d})$ derived according to models (1) and (3)-(4), respectively, for eight observations generated at random with $F=0.010 \mathrm{~mm}$ and $\sigma_{M}=0.002 \mathrm{~mm}$.

Applying Bayes' theorem

$$
\begin{aligned}
p(F \mid \boldsymbol{d}) & \propto p(\boldsymbol{d} \mid F) p(F) \\
& \propto \frac{1}{F^{m+1}} \prod_{i=1}^{m} I\left(d_{i}, \sigma_{M},-F, F\right) .
\end{aligned}
$$

The integrating constant associated with the distribution, and the distribution mean, variance and quartiles, etc., can be determined using numerical integration [19]. This distribution can be used to make inferences about $F$, taking into account the measurement uncertainty associated with the evaluated residual distances, according to the model in (3)-(4).

Figure 1 shows posterior distributions for eight observations generated according to model (3)-(4) with $F=0.010 \mathrm{~mm}$ and $\sigma_{M}=0.002 \mathrm{~mm}$. For this data set $F_{0}=\max _{i} d_{i}=0.0094 \mathrm{~mm}$. The distribution $p(F \mid \boldsymbol{f})$, Figure 1 (solid curve), is the Pareto distribution $p\left(F \mid F_{0}=\right.$ $0.0094, m=8$ ) derived from the data and ignores measurement uncertainty. The mean of this distribution is $m F_{0} /(m-1)=0.0107 \mathrm{~mm}$. The estimate $\hat{F}$ given by $(5)$ is $\hat{F}=0.0110 \mathrm{~mm}$.

The distribution $p(F \mid \boldsymbol{d})$, Figure 1 (dotted curve), is derived according to (8). While the distribution $p(F \mid \boldsymbol{f})$ assigns no probability mass to values of $F<F_{0}$, the distribution $p(F \mid \boldsymbol{d})$ takes into account the measurement uncertainty to provide a smoother, Gaussian-like cut off. The mean of the distribution $p(F \mid \boldsymbol{d})$ is $0.0102 \mathrm{~mm}$.

\subsection{Asymmetric rectangular distribution}

Now suppose $f_{i}, i=1, \ldots, m$ are draws from a rectangular distribution $\mathrm{R}(A-F, A+F)$,

$$
f_{i} \in \mathrm{R}(A-F, A+F),
$$

so that

$$
p(\boldsymbol{f} \mid F, A, m)=\frac{1}{2^{m}} \frac{1}{F^{m}} .
$$

Let $U=\max _{i} f_{i}, L=\min _{i} f_{i}, F_{0}=(U-L) / 2$ and $A_{0}=$ $(U+L) / 2$. If the joint prior distribution $p(F, A)$ for $F$ and $A$ is $p(F, A) \propto 1 / F$, then

$$
p\left(F, A \mid F_{0}, U_{0}\right)=\frac{m(m-1)}{2} F_{0}^{m-1} \frac{1}{F^{m+1}},
$$

if $F \geq F_{0}$ and

$$
A \in\left[A_{0}-\left(F-F_{0}\right), A_{0}+\left(F-F_{0}\right)\right],
$$

and is zero elsewhere. The marginalised posterior distributions are

$$
p\left(F \mid F_{0}, A_{0}\right)=m(m-1)\left(F-F_{0}\right) F_{0}^{m-1} \frac{1}{F^{m+1}},
$$

if $F \geq F_{0}$, and is zero elsewhere, and

$$
p\left(A \mid F_{0}, A_{0}\right)=\frac{m-1}{2} F_{0}^{m-1}\left(\frac{1}{F_{0}+\left|A-A_{0}\right|}\right)^{m} .
$$

The means of these two distributions are $m F_{0} /(m-2)$, $m>2$, and $A_{0}$, respectively.

\subsubsection{Incorporating measurement uncertainty}

Suppose that

$$
d_{i}=f_{i}+\epsilon_{i}
$$

with

$$
f_{i} \in \mathrm{R}(A-F, A+F), \quad \epsilon_{i} \in \mathrm{N}\left(0, \sigma_{M}^{2}\right) .
$$

Given $\boldsymbol{d}$ and $\sigma_{M}$, the quantity $\hat{F}$ given in (5) is an estimate of $F$, also. Using a Bayesian approach, we have

$$
p(F, A \mid \boldsymbol{d}) \propto \frac{1}{F^{m+1}} \prod_{i=1}^{m} I\left(d_{i}, \sigma_{M}, A-F, A+F\right),
$$

where the integrals $I\left(d_{i}, \sigma_{M}, A-F, A+F\right)$ are evaluated using (7). The integration constant, marginal distributions $p(F \mid \boldsymbol{d}), p(A \mid \boldsymbol{d})$, means, etc., can be determined using numerical quadrature in one or two dimensions.

Figure 2 shows posterior distributions for eight observations generated according to model (3)-(4) with $F=0.010 \mathrm{~mm}$ and $\sigma_{M}=0.002 \mathrm{~mm}$. For this data set, $F_{0}=\left(\max _{i} d_{i}-\min _{i} d_{i}\right) / 2=0.0087 \mathrm{~mm}$ and $A_{0}=$ $\left(\max _{i} d_{i}+\min _{i} d_{i}\right) / 2=-0.0007 \mathrm{~mm}$. The distribution $p(F \mid \boldsymbol{f})$, Figure 2 (solid curve), is the distribution defined by $F_{0}$ and $A_{0}$ in (10) and ignores measurement uncertainty. Compared with $p(F \mid \boldsymbol{f})$ in Figure 1, the cut-off is less abrupt due to the fact that $A$ is also uncertain. The mean of this distribution is $m F_{0} /(m-2)=0.0115 \mathrm{~mm}$. The distribution $p(F \mid \boldsymbol{d})$, Figure 2 (dotted curve), is calculated from (14) and numerical marginalisation. The mean of this distribution is $0.0112 \mathrm{~mm}$. 


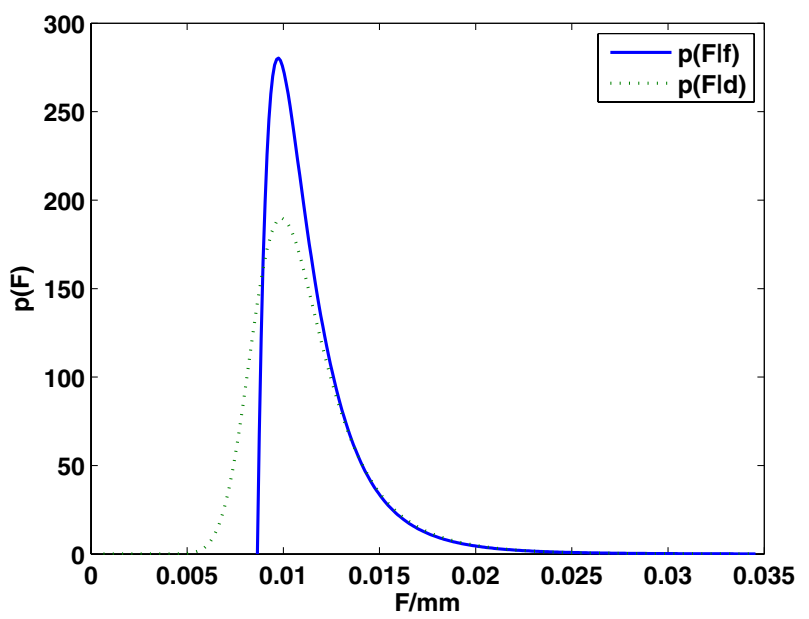

Fig. 2. Posterior densities $p(F \mid \boldsymbol{f})$ and $p(F \mid \boldsymbol{d})$ derived according to models (9), and (12)-(13), respectively, for eight observations generated at random with $F=0.0100 \mathrm{~mm}$, $A=0.0000 \mathrm{~mm}$ and $\sigma_{M}=0.002 \mathrm{~mm}$.

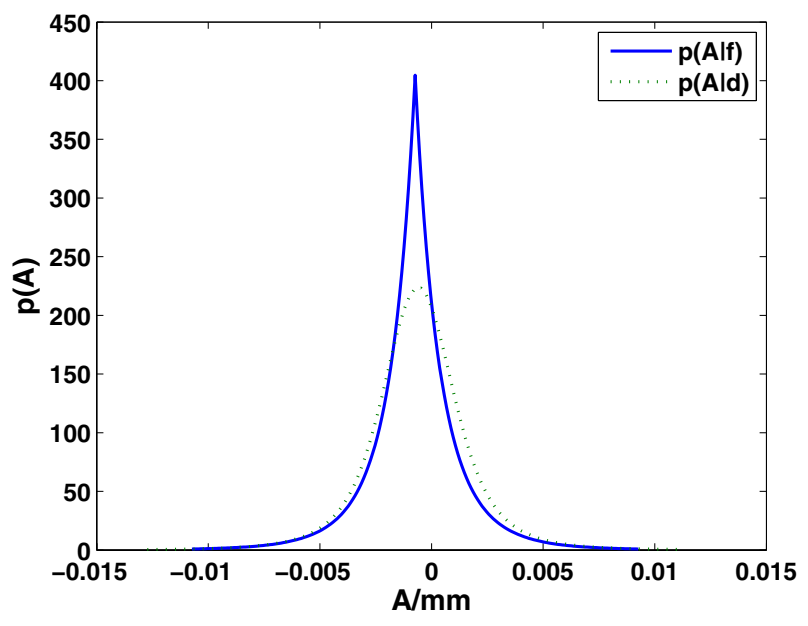

Fig. 3. Posterior densities $p(A \mid \boldsymbol{f})$ and $p(A \mid \boldsymbol{d})$ derived according to models (9), and (12)-(13), respectively, for eight observations generated at random with $F=0.0100 \mathrm{~mm}$, $A=0.0000 \mathrm{~mm}$ and $\sigma_{M}=0.002 \mathrm{~mm}$.

\section{Spatially correlated form error models}

The models and inference approaches for estimating form error discussed in the previous section do not take into account the locations of the points on the surface $s_{i}$ at which the form error is estimated. In particular, the form errors are modelled as being independently distributed. However, our experience suggests that for many if not all applications that the form error is likely to vary somewhat smoothly and that the form error at a location is a reasonable guide to the size of the form error at nearby locations. We use a Gaussian Process (GP) approach [20] to model this spatial correlation. A simple implementation is given below.

Suppose that $f$ and $f^{\prime}$ are the form errors associated with locations $s$ and $\boldsymbol{s}^{\prime}$, respectively. We consider covari- ance models of the form

$$
\operatorname{cov}\left(f, f^{\prime}\right)=k\left(s, s^{\prime}\right),
$$

where the covariance of $f$ with $f^{\prime}$ depends on the spatial locations, $s$ and $\boldsymbol{s}^{\prime}$ through the kernel $k$. For example, we can choose a squared exponential kernel

$$
k\left(\boldsymbol{s}, \boldsymbol{s}^{\prime} \mid \boldsymbol{\sigma}_{F}\right)=\sigma^{2} \exp \left\{-\frac{1}{2 \lambda^{2}}\left\|\boldsymbol{s}-\boldsymbol{s}^{\prime}\right\|^{2}\right\}+\sigma_{0}^{2} \delta\left(\boldsymbol{s}, \boldsymbol{s}^{\prime}\right) .
$$

Here, $\boldsymbol{\sigma}_{F}=\left(\sigma, \sigma_{0}, \lambda\right)^{\mathrm{T}}$, where $\sigma^{2}$ represents the variance of the form error over the complete surface, $\lambda$ defines the length scale for the spatial correlation, $\sigma_{0}^{2}$ the variance of the form error at very short length scales due to roughness effects, for example, and $\delta\left(s, s^{\prime}\right)=1$ if $s=s^{\prime}$ and is zero otherwise. The idea of a GP approach is to use measurements of the form error at points on the surface to estimate the form error at unmeasured points on the strength of the spatial correlation. Let $\boldsymbol{f}_{1}$ be the form errors $f_{i}$ at spatial locations $\boldsymbol{s}_{i}, i \in I_{1}$ and $\boldsymbol{f}_{2}$ be those at locations at $s_{i}, i \in I_{2}$. Before any measurement is made, our prior model is that

$$
\left[\begin{array}{l}
\boldsymbol{f}_{1} \\
\boldsymbol{f}_{2}
\end{array}\right] \sim \mathrm{N}(\mathbf{0}, V)
$$

where variance matrix $V$ is defined by the correlation kernel

$$
V_{i j}=k\left(\boldsymbol{s}_{i}, \boldsymbol{s}_{j}\right) \approx k\left(\boldsymbol{x}_{i}, \boldsymbol{x}_{j}\right) .
$$

We determine the Cholesky factorisation [21] of $V$ so that $V=L L^{\mathrm{T}}$ and partition $L$ corresponding to the index sets $I_{k}, k=1,2$ :

$$
L=\left[\begin{array}{ll}
L_{11} & \\
L_{21} & L_{22}
\end{array}\right]
$$

Hence the prior information (15) is equivalent to

$$
\left[\begin{array}{l}
\boldsymbol{f}_{1} \\
\boldsymbol{f}_{2}
\end{array}\right]=\left[\begin{array}{ll}
L_{11} & \\
L_{21} & L_{22}
\end{array}\right]\left[\begin{array}{l}
\boldsymbol{e}_{1} \\
\boldsymbol{e}_{2}
\end{array}\right]
$$

with

$$
\left[\begin{array}{l}
\boldsymbol{e}_{1} \\
\boldsymbol{e}_{2}
\end{array}\right] \sim \mathrm{N}(\mathbf{0}, I)
$$

Now suppose we record observations $\boldsymbol{d}_{1}$ of the form errors $\boldsymbol{f}_{1}$ according to the model

$$
\boldsymbol{d}_{1}=\boldsymbol{f}_{1}+\boldsymbol{\epsilon}_{1}, \quad \boldsymbol{\epsilon}_{1} \in \mathrm{N}\left(\mathbf{0}, \sigma_{M}^{2}\right) .
$$

The prior information about $\boldsymbol{e}_{1}$ in (16) can be combined with the measurement information (17) about $\boldsymbol{f}_{1}=L_{11} \boldsymbol{e}_{2}$ to obtain updated information

$$
\boldsymbol{e}_{1} \mid \boldsymbol{d}_{1} \sim \mathrm{N}\left(\hat{\boldsymbol{e}}_{1}, V \boldsymbol{e}_{1}\right)
$$

where $\hat{\boldsymbol{e}}_{1}$ is the solution of the least squares system $A e_{1}=c$, with

$$
A=\left[\begin{array}{c}
L_{11} / \sigma_{M} \\
I
\end{array}\right], \quad \boldsymbol{c}=\left[\begin{array}{c}
\boldsymbol{d}_{1} / \sigma_{M} \\
\mathbf{0}
\end{array}\right],
$$




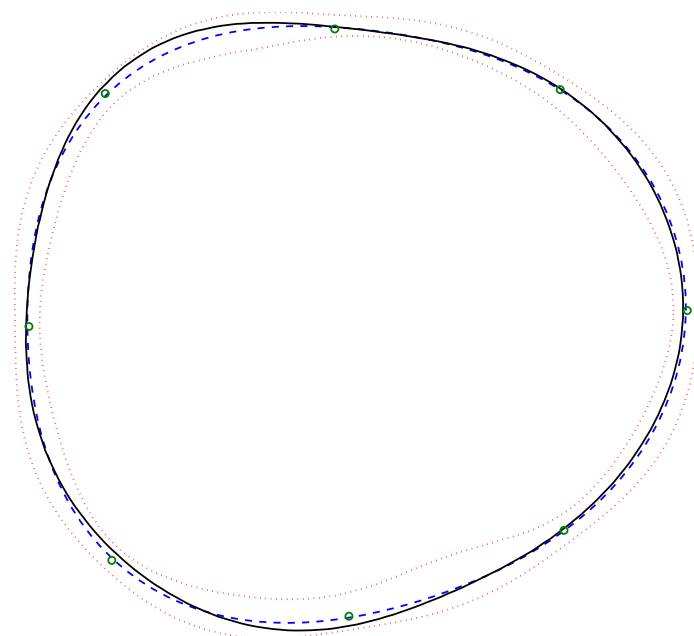

Fig. 4. Representation of the form of a circular artefact (solid curve), measured coordinates (circles), the best estimate of the form (dashes), reconstructed on the basis of the measured data and the spatial correlation, the uncertainty band representing plus and minus two standard deviations for the form (dots).

and

$$
V_{\boldsymbol{e}_{1}}=\left(A^{\mathrm{T}} A\right)^{-1} .
$$

Given $\boldsymbol{d}_{1}$, the posterior distribution for $\boldsymbol{f}_{1} \mid \boldsymbol{d}_{1}$ is

$$
\boldsymbol{f}_{1} \mid \boldsymbol{d}_{1} \sim \mathrm{N}\left(L_{11} \hat{\boldsymbol{e}}_{1}, L_{11} V_{\boldsymbol{e}_{1}} L_{11}^{\mathrm{T}}\right)
$$

The measurements $\boldsymbol{d}_{1}$ provide no information about $\boldsymbol{e}_{2}$, so $\boldsymbol{e}_{2} \mid \boldsymbol{d}_{1} \sim \mathrm{N}(\mathbf{0}, I)$, as before, so that

$$
\boldsymbol{f}_{2} \mid \boldsymbol{d}_{1} \sim \mathrm{N}\left(L_{21} \hat{\boldsymbol{e}}_{1}, V_{2}\right)
$$

with $V_{2}=L_{21} V_{\boldsymbol{e}_{1}} L_{21}^{\mathrm{T}}+L_{22} L_{22}^{\mathrm{T}}$. In this way, the measurements $\boldsymbol{d}_{1}$ are used to provide updated estimates of $\boldsymbol{f}_{2}$ along with the associated variance matrix.

A posterior distribution for the form error $F_{E}$ can be determined using a Monte Carlo approach by sampling $\boldsymbol{f}_{q} \in \mathrm{N}\left(L_{21} \hat{\boldsymbol{e}}_{1}, V_{2}\right)$ and then evaluating

$$
F_{E, q}=\max _{i} f_{i, q}-\min _{i} f_{i, q} .
$$

The sampled vector of form errors $\boldsymbol{f}_{q}$ can be determined as follows. If $V_{2}$ has Cholesky factorisation $V_{2}=L_{2} L_{2}^{\mathrm{T}}$ then we draw $\boldsymbol{e}_{q} \in \mathrm{N}(\mathbf{0}, I)$ and set

$$
\boldsymbol{f}_{q}=L_{21} \hat{\boldsymbol{e}}_{1}+L_{2} \boldsymbol{e}_{q} .
$$

Figure 4 shows the form of a circular artefact (solid curve), measured coordinates (circles), the best estimate of the form (dashes), reconstructed on the basis of the measured data and the spatial correlation, the uncertainty band representing plus and minus two standard deviations for the form (dots). The uncertainty band narrows around the measurement points. The size of the form error has been greatly magnified to show the effects.

Figure 5 is a histogram representation of the distribution for $F_{E} / 2$, derived using Monte Carlo samples for data associated with Figure 4 . The mean of the sampled $F_{E, q} / 2$ was $0.0098 \mathrm{~mm}$ with an associated standard deviation of $0.0015 \mathrm{~mm}$.

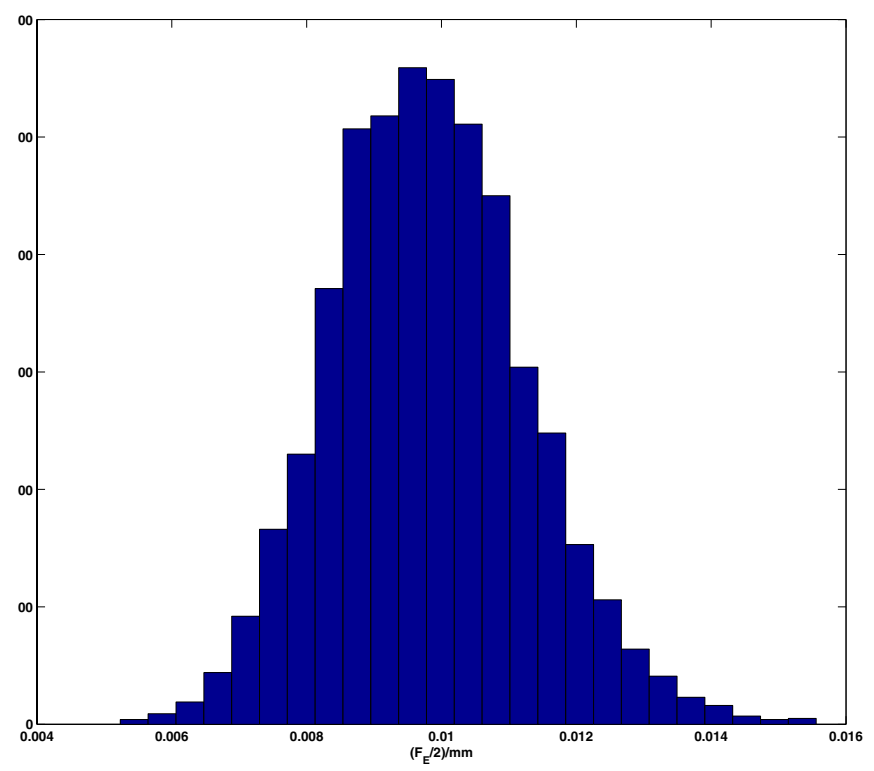

Fig. 5. Sampled form error $F_{E, q} / 2$ calculated as in equations (18) and (19) for data shown in Figure 4.

\section{Discussion}

The approaches described in Sections 2 and 3 above have been concerned with evaluating the uncertainty associated the estimate of form error on the basis of measured data. The main aim has been to provide evaluated uncertainties that make modest computation demand both in terms of computation time and algorithmic complexity. The approaches in Section 2 have involved distributions that can be defined analytically or involve numerical integration along one or two axes. The techniques described in Section 3 involving spatial correlation require computational approaches that one uses regularly in least squares parameter estimation [22]. However, the approaches do rely on a number of assumptions, as discussed below.

The approaches assume that the measurement system is well-characterised so that $\sigma_{M}$ is known and that the measurements are statistically independent, e.g., $\boldsymbol{d} \in$ $\mathrm{N}\left(\boldsymbol{f}, \sigma_{M}^{2} I\right)$. The calculations associated with rectangular distribution in Section 2 make significant use of these assumptions in order to derive semi-analytical expressions. A more general approach is to assume that

$$
\boldsymbol{d} \sim \mathrm{N}\left(\boldsymbol{f}, V\left(\boldsymbol{\sigma}_{M}\right)\right)
$$

where the variance matrix $V\left(\boldsymbol{\sigma}_{M}\right)$ associated with the measured results $\boldsymbol{d}$ depends on statistical parameters $V\left(\boldsymbol{\sigma}_{M}\right)$ associated with aspects of the measurement system. For example, dealing with data gathered by a coordinate measuring machine $(\mathrm{CMM})$ the parameters $\boldsymbol{\sigma}_{M}$ could characterise random effects and also scale, squareness and other kinematic errors associated with the CMM [7,8,23]. Similarly, for the GP approach, the variance matrix $V\left(\boldsymbol{\sigma}_{F}\right)$ specifying the spatial correlation of the form error depends on statistical parameters $\boldsymbol{\sigma}_{F}$. Rather than regarding these parameters as fixed, it may be more realistic to regard them as additional hyper-parameters for where 
there is prior information in the form of prior distributions and to use the measurement data to provide updated distributions for these parameters. In a Gaussian process (GP) setting, there is algorithmic machinery to deal with this case and so, if necessary, the simple approach described in Section 3 could be extended to take account this more general model. For such generalisations, the semianalytical approaches described in Section 2 would have to give way to more numerical procedures such as Markov chain Monte Carlo methods [15,24].

The approaches also ignore that the fact that the estimates $d_{i}$ of the form error $f_{i}$ at the points $s_{i}$ on the surface are determined as a result of surface fitting and are therefore subject to a correlating effect associated with the fitting. The GP approaches can accommodate this fact easily if the surface fitting is according to a least squares criterion (or a generalised least squares approach [25]) but there are considerable complications if a Chebyshev (minimum zone) approach is implemented.

\section{Summary and concluding remarks}

This paper has been concerned with the evaluating the uncertainty associated with the assessment of form error taking into account measurement uncertainty and sampling effects. We have described simple, semi-analytical approaches based on rectangular distributions and slightly more comprehensive Gaussian process (GP) models that take into account the likely spatial correlation of form error. These methods are aimed at deriving uncertainty estimates without excessively complicated computational machinery. The GP model can be extend quite considerably, especially in an off-line context, where it is required to design a measurement strategy that will be evaluate form error to within pre-assigned.

Acknowledgements. This work was funded by the UK's National Measurement Office programme for Mathematics and Modelling.

\section{References}

1. S.J. Ahn, E. Westkämper, W. Rauh, Orthogonal distance fitting of parametric curves and surfaces, in Algorithms for Approximation IV, edited by J. Levesley, I.J. Anderson, J.C. Mason (University of Huddersfield, 2002), pp. $122-129$

2. G.T. Anthony, H.M. Anthony, B. Bittner, B.P. Butler, M.G. Cox, R. Drieschner, R. Elligsen, A.B. Forbes, H. Groß, S.A. Hannaby, P.M. Harris, J. Kok, Reference software for finding Chebyshev best-fit geometric elements, Precis. Eng. 19, 28-36 (1996)

3. P.T. Boggs, R.H. Byrd, R.B. Schnabel, A stable and efficient algorithm for nonlinear orthogonal distance regression, SIAM J. Sci. Statist. Comput. 8, 1052-1078 (1987)

4. K. Carr, P. Ferreira, Verification of form tolerances part I: Basic issues, flatness and straightness, Precis. Eng. 17, 131-143 (1995)
5. K. Carr, P. Ferreira, Verification of form tolerances part II: Cylindricity and straightness of a median line, Precis. Eng. 17, 144-156 (1995)

6. A.B. Forbes, Least squares best fit geometric elements, in Algorithms for Approximation II, edited by J.C. Mason, M.G. Cox (Chapman \& Hall, London, 1990), pp. 311-319

7. A.B. Forbes, Surface fitting taking into account uncertainty structure in coordinate data, Meas. Sci. Technol. 17, 553-558 (2006)

8. A.B. Forbes, Uncertainty evaluation associated with fitting geometric surfaces to coordinate data, Metrologia 43, S282-S290 (2006)

9. A.B. Forbes, H.D. Minh, Form assessment in coordinate metrology, in Approximation Algorithms for Complex Systems, edited by E.H. Georgoulis, A. Iske, J. Levesley, Springer Proceedings in Mathematics (Springer-Verlag, Heidelberg, 2011), Vol. 3, pp. 69-90

10. H.-P. Helfrich, D. Zwick, $\ell_{1}$ and $\ell_{\infty}$ Fitting of Geometric Elements (2002), pp. 162-169

11. X. Jiang, X. Zhang, P.J. Scott, Template matching of freeform surfaces based on orthogonal distance fitting for precision metrology, Meas. Sci. Technol. 21, 045101 (2010)

12. G. Moroni, S. Petrò, Geometric tolerance evaluation: a discussion on minimum zone fitting algorithms, Precis. Eng. 32, 232-237 (2008)

13. D. Sourlier, W. Gander, A new method and software tool for the exact solution of complex dimensional measurement problems, in Advanced Mathematical Tools in Metrology II, edited by P. Ciarlini, M.G. Cox, F. Pavese, D. Richter (World Scientific, Singapore, 1996), pp. 224-237

14. G.E.P. Box, G.C. Tiao, Bayesian Inference in Statistical Analysis (Wiley, New York, Wiley Classics Library, edition 1992, edition 1973)

15. A. Gelman, J.B. Carlin, H.S. Stern, D.B. Rubin, Bayesian Data Analysis, 2nd edn. (Chapman \& Hall/CRC, Boca Raton, 2004)

16. H.S. Migon, D. Gamerman, Statistical Inference: an Integrated Approach (Arnold, London, 1999)

17. D.S. Sivia, Data Analysis: a Bayesian Tutorial (Clarendon Press, Oxford, 1996)

18. M. Evans, N. Hastings, B. Peacock, Statistical distributions (Wiley, 2000)

19. W.H. Press, B.P. Flannery, S.A. Teukolsky, W.T. Vetterling, Numerical Recipes: the Art of Scientific Computing (Cambridge University Press, Cambridge, 1989), www.nr.com

20. C.E. Rasmussen, C.K.I. Williams, Gaussian Processes for Machine Learning (MIT Press, Cambridge, 2006)

21. G.H. Golub, C.F. Van Loan, Matrix Computations, 3rd edn. (John Hopkins University Press, Baltimore, 1996)

22. A.B. Forbes, Parameter estimation based on least squares methods, in Data Modeling for Metrology and Testing in Measurement Science, edited by F. Pavese, A.B. Forbes, (Birkhäuser-Boston, New York, 2009), pp. 147-176

23. G. Zhang, R. Ouyang, B. Lu, R. Hocken, R. Veale, A. Donmez, A displacement method for machine geometry calibration, Ann. CIRP 37, 515-518 (1988)

24. D. Gamerman, Markov chain Monte Carlo: Stochastic Simulation for Bayesian Inference (Taylor \& Francis, New York, 1997)

25. A.B. Forbes, Structured nonlinear Gauss-Markov Problems, in Algorithms for Approximation $V$, edited by A. Iske, J. Levesley (Springer, Berlin, 2006), pp. 167-186 